\title{
BMJ Global Health Effectiveness, safety and acceptability of medical abortion at home versus in the clinic: a systematic review and meta- analysis in response to COVID-19
}

To cite: Gambir K, Garnsey C, Necastro KA, et al. Effectiveness, safety and acceptability of medical abortion at home versus in the clinic: a systematic review and meta-analysis in response to COVID-19. BMJ Global Health 2020;5:e003934. doi:10.1136/ bmjgh-2020-003934

Handling editor Seye Abimbola

- Additional material is published online only. To view please visit the journal online (http://dx.doi.org/10.1136/ bmjgh-2020-003934).

Received 9 September 2020 Revised 25 November 2020 Accepted 26 November 2020

Check for updates

C Author(s) (or their employer(s)) 2020. Re-use permitted under CC BY-NC. No commercial re-use. See rights and permissions. Published by BMJ.

${ }^{1}$ Poverty, Gender and Youth Program, Population Council, New York, New York, USA ${ }^{2}$ Department of Psychiatry, Massachusetts General Hospital Boston, Massachusetts, USA ${ }^{3}$ The GIRL Center, Population Council, New York, New York, USA

Correspondence to Katherine Gambir; kgambir@gmail.com

\section{ABSTRACT}

Background Increased access to home-based medical abortion may offer women a convenient, safe and effective abortion method, reduce burdens on healthcare systems and support social distancing during the COVID-19 pandemic. Home-based medical abortion is defined as any abortion where mifepristone, misoprostol or both medications are taken at home.

Methods A systematic review and meta-analysis of randomised controlled trials (RCTs) and non-randomised studies (NRSs) were conducted. We searched databases from inception to 10 July 2019 and 14 June 2020. Successful abortion was the main outcome of interest. Eligible studies were RCTs and NRSs studies with a concurrent comparison group comparing home versus clinic-based medical abortion. Risk ratios (RRs) and their 95\% Cls were calculated. Estimates were calculated using a random-effects model. We used the Grading of Recommendations Assessment, Development and Evaluation approach to assess risk of bias by outcome and to evaluate the overall quality of the evidence.

Results We identified 6277 potentially eligible published studies. Nineteen studies (3 RCTs and 16 NRSs) were included with 11576 women seeking abortion up to 9 weeks gestation. Neither the RCTs nor the NRS found any difference between home-based and clinic-based administration of medical abortion in having a successful abortion (RR 0.99, 95\% Cl 0.98 to $1.01, \mathrm{I}^{2}=0 \%$; RR 0.99 , $95 \% \mathrm{Cl} 0.97$ to $1.01, \mathrm{I}^{2}=52 \%$, respectively). The certainty of the evidence for the 16 NRSs was downgraded from low to very low due to high risk of bias and publication bias. The certainty of the evidence for the three RCTs was downgraded from high to moderate by one level for high risk of bias.

Conclusion Home-based medical abortion is effective, safe and acceptable to women. This evidence should be used to expand women's abortion options and ensure access to abortion for women during COVID-19 and beyond.

PROSPERO registration number CRD42020183171.

\section{INTRODUCTION}

Of the estimated 55 million abortions that occur worldwide each year, 25 million (45\%)
Key questions

What is already known?

- In 2011, Ngo et al reviewed evidence from nine nonrandomised studies (NRSs) which suggested that there is no difference in the effectiveness or acceptability of home-based medical abortion compared to clinic-based medical abortion (OR $0.8,95 \% \mathrm{Cl} 0.5$ to $\left.1.5, I^{2} 69.4 \%\right)$

- Since 2011, the evidence on home-based medical abortion has grown, particularly with the publication of three randomised controlled trials (RCTs), but access to home-based medical abortion remains limited, and restrictions on travel and movement put in place due to COVID-19 has further complicated access to abortion worldwide.

- A recent Cochrane review from 16 NRSs and 2 RCTs found no difference in effectiveness between selfadministered and provider-administered abortions (risk ratio $0.99,95 \% \mathrm{Cl} 0.97$ to 1.01 ); but this review did not analyse the location of the procedure and the quality of evidence was reported as moderate for the RCTs and very low for the NRSs.

What are the new findings?

- Home-based medical abortion is effective, safe and acceptable to women.

- There is no difference in the effectiveness or safety of medical abortion completed at home or in a clinic.

- Home-based medication abortion was highly acceptable to women, supporting existing evidence that women may prefer home administration to clinic-based care for reasons related to control and privacy, preferences that may be more pronounced due to concerns around spreading or contracting novel coronavirus disease 2019 (COVID-19).

are unsafe, with most unsafe abortions occurring in low-resource settings. ${ }^{1}$ The expanded use of medical abortion worldwide, and particularly in low-resource settings, has been linked to increasing rates of safe abortion, and a decline in the morbidity and mortality associated with unsafe abortion, ${ }^{2-4}$ yet access to medication abortion continues 


\section{Key questions}

What do the new findings imply?

- Home-based medical abortion may be an important strategy to offer women a convenient, safe and effective abortion method, reduce burdens on healthcare systems and support social distancing policies during the COVID-19 pandemic and beyond.

- Researchers, policy- makers and practitioners must continue to work to develop, evaluate and expand access to novel and innovative service delivery models such as telemedicine for medical abortion, pharmacist and community health worker provision of medical abortion and home-based medical abortion with hotline support.

- The update to the evidence comes at a time of global crisis, policymakers should use the evidence presented in this review alongside national and international guidelines on medical abortion to expand home-based abortion care during and following the pandemic.

to be limited by numerous restrictions. ${ }^{5-9}$ Health systems worldwide have been burdened by the COVID-19 global pandemic ${ }^{10-12}$ and the necessary but drastic restrictions on movement that governments worldwide have instated in response to the pandemic further complicate access to abortion.

Expanding access to home-based abortion care is an important strategy for protecting abortion access while slowing the spread of coronavirus and reducing burdens on healthcare systems, especially in settings where capacity and workforces are already strained and limited. Home-based medical abortion may involve the pregnant person taking both mifepristone and misoprostol at home or misoprostol only at home after taking mifepristone at a clinic. Either way, the model requires fewer clinic visits, alleviating burdens on healthcare facilities and providers and limiting points of contact. ${ }^{713}$ In this review, women who took either medication at home were classified as belonging to the home-based group. That is, women who took mifepristone and misoprostol at home or took only misoprostol at home after taking mifepristone in the clinic were classified as home-based participants. However, none of the eligible studies compared women who took both mifepristone and misoprostol at home to participants who took both medications at the clinic.

This systematic review and meta-analysis aims to compare the effectiveness, safety, and acceptability of home-based medical abortion versus clinic-based irrespective of regimen for women of any age seeking abortion services in any setting. This is an update to a review conducted in 2011 by Ngo et $a l^{14}$ and includes the most-up-to-date evidence for health officials and other public health decision makers across the globe to make evidence-informed decisions on home-based medical abortion guidelines during and following the pandemic. The recent Cochrane review from 16 non-randomised studies (NRSs) and two randomised controlled trials (RCTs) that compared the effectiveness between selfadministered and provider-administered abortions (risk ratio (RR) $0.99,95 \%$ CI 0.97 to 1.01 ) did not analyse the location of the procedure. However, abortion policies and clinical guidance are very precise on who can provide the procedure and where it takes place; therefore, it is important to provide this level of evidence for policy makers and clinicians. Given the number of new studies that have been published in the past decade, the persistent restrictions on medical abortion, the need to expand people's choices, and the importance of access to home-based medical abortion in the context of the COVID-19 crisis, it is critically important to assess the most up to date evidence on home-based abortion and provide these data to health officials to formulate guidelines during and following the pandemic.

\section{METHODS}

\section{Search strategy and selection criteria}

We conducted a systematic review and meta-analysis that updates the home-based versus clinic-based systematic review by Ngo et al. ${ }^{14}$ The search strategy used in this review is an update to the search conducted in July 2019 by Gambir et al for a review of the comparative effectiveness, safety, and acceptability of self-administered vs provider administered medical abortion review. ${ }^{15}$ All published and unpublished RCTs and NRSs were eligible for inclusion if the review if they: (1) had a comparison between home-based versus clinic-based medical abortion; (2) compared the safety, or effectiveness, or both of women in both groups (3) assessed outcomes prospectively and (4) included a sample of women of reproductive ages (15-49) who were able to provide informed consent and were seeking an induced abortion in any clinical setting. Studies that compared home-based and clinic-based administration of medical abortion, irrespective of the drug regimens, routes of administration, doses used and person who administered the $\operatorname{drug}(\mathrm{s})$ were considered eligible. Studies were excluded if they recruited women with missed or incomplete abortions, or women who had experienced intrauterine fetal deaths.

We searched without language restrictions. We searched MEDLINE, Embase, CINAHL, LILACS, ClinicalTrials. gov, Google Scholar and PubMed from inception to 14 June 2020. We searched Cochrane Central Register of Controlled Trials, POPLINE and WHO ICTRP from inception to 10 July 2019 (update searches were not performed for these databases as they were not available at the time of the update). We also searched the websites of Marie Stopes International, Ipas, Gynuity Health Projects, Population Council and the International Consortium for Medical Abortion and hand-searched reference lists of relevant trials and systematic reviews returned in the electronic search. The full search strategy is available in online supplemental appendix 1.

Three reviewers (KG, KAN and CG) independently screened titles and abstracts returned by the search and retrieved full texts of all studies that were potentially eligible for inclusion. They independently examined all full-text articles for inclusion in the review using the 
predetermined inclusion and exclusion criteria. Disagreements and concerns about eligibility were resolved by discussion. Two studies ${ }^{1617}$ were translated from French to English.

The protocol for this review was published on 5 May 2020, DOI: https://doi.org/10.7910/DVN/NPG1GK/ $\mathrm{E}^{\mathrm{XNMJ}}{ }^{18}$

\section{Data analysis}

Three independent reviewers (KG, KAN, CG) extracted data from eligible studies using a data abstraction form the review authors designed and piloted. Reviewers requested data from study authors via email as needed. The proportion of women with successful abortion is the primary outcome for the review. For all studies, successful abortion was defined as a complete uterine evacuation without the need for surgical intervention. In each study, successful abortion was determined by the healthcare provider during the follow-up visit. We also extracted data on study design (including details of treatments provided to the intervention and comparison groups), homebased participants and comparison groups recruited and analysed, population characteristics, duration and prevalence of side effects, acceptability of medication abortion, companionship during abortion and compliance with medication abortion protocol and follow-up.

The primary measure of effect assessed in this review is the RR of having a successful home-based medical abortion compared with having a successful clinicbased medical abortion, and the $95 \%$ CI of the RR. We calculated the RR of having a successful abortion using the number of women recruited for each study and an intention-to-treat approach. When intention-to-treat data were not available, we used a per protocol approach. We also present RRs and 95\% CIs for variables related to complications and acceptability. We reported test statistics and narrative summaries for outcomes of side effects, companionship during home administration and compliance with medical abortion protocol.

Two review authors (KG and KAN) independently assessed eligible RCTs using Cochrane risk of bias tools for RCTs and NRSs using ROBINS-I tool to assess bias at the outcome level for our primary outcome of successful abortion. ${ }^{19}$ We used Grading of Recommendations Assessment, Development and Evaluation (GRADE) criteria to determine the overall quality of evidence. ${ }^{19}$ We downgraded the quality of evidence of RCTs from a starting rating of 'high certainty' by one level for serious concerns or by two levels for very serious concerns about risk of bias, inconsistency, indirectness, imprecision and publication bias. For NRSs, we used the same downgrade approach, but started with a baseline rating of 'low certainty' due to the lack of randomisation. Two review authors (KG and KAN) worked independently to rate the overall evidence quality (eg, high, moderate, low or very low) and disagreements were resolved by discussion.

We synthesised effectiveness in a meta-analysis using a random effects model to create RRs and their 95\% CIs.
This model was selected a priori to incorporate the effect of trial heterogeneity among prospective studies from different settings. ${ }^{14}$ The meta-analysis was conducted using standard weighted (by SE of estimate) linear regression using RevMan Web. ${ }^{20}$ We constructed forest plots showing the RRs and their 95\% CI for the primary outcome of successful abortion. We analysed RCTs and NRSs separately when summarising the effect sizes of successful abortion, and for secondary outcomes. We performed statistical analysis using RevMan Web. ${ }^{20}$

We examined statistical heterogeneity, especially when there was any variation in the direction of the effect. Statistical heterogeneity was assessed with $\chi^{2}$ and $\mathrm{I}^{2}$ statistics with a cut-off point of $\mathrm{p}<0.10$ to indicate statistical heterogeneity and we used the $\mathrm{I}^{2}$ statistic to quantify heterogeneity. We did not find substantial heterogeneity in our meta-analysis, and therefore, we did not explore possible explanations in subgroup analysis.

We did not conduct a sensitivity analysis to assess the effect of the risk of bias of the studies included in the main effects analysis, because of the results of our risk of bias assessments.

\section{Patient and public involvement}

Given this is a systematic review of available global evidence, patients who participated in the studies presented were not involved in the review process.

\section{RESULTS}

We identified 2643 non-duplicate citations through database searching, assessed 41 full-text articles for eligibility and included 19 studies in the meta-analysis and systematic review (figure 1). The updated June 2020 search yielded one additional study Song 2018 retrieved from PubMed. No data from unpublished studies were identified. One included study collected data in two locations-Tunisia and Vietnam and each location is classified as a separate study and data is presented separately in our review. All included studies reported on our primary outcome of successful abortion. Three studies were RCTs, and included data from 1452 women. ${ }^{21-23}$ Sixteen studies were NRSs and included data from 10124 women. ${ }^{16}{ }^{17} 24-36$ Sixteen studies were conducted in lowto-middle resource settings (Bangladesh, ${ }^{25}{ }^{26}$ India, ${ }^{28} 3034$ Nepal, ${ }^{2231}$ China, ${ }^{2123}$ Vietnam, ${ }^{323335}$ Tunisia, ${ }^{2935}$ Nigeria,${ }^{36}$ Albania $^{27}$ and Turkey ${ }^{24}$ ). Two studies were conducted in France, a high resource setting. ${ }^{16} 17$ Table 1 provides more details on study characteristics.

The mean age of study participants ranged from 24.3 years $^{36}$ to 32.2 years of age. ${ }^{35}$ Participants' maximum gestational age was under 9 weeks for 14 studies, ${ }^{16} 17212324$ 27-29 31-35 and up to and including 9 weeks for the other five studies. ${ }^{22} 25263036$ Statistically significant differences between participants in the homebased and clinic-based groups were reported on variables including primigravida, parity, age and gestational age in six studies. ${ }^{16}{ }^{1728-30} 32$ Two studies ${ }^{35}$ did not compare 

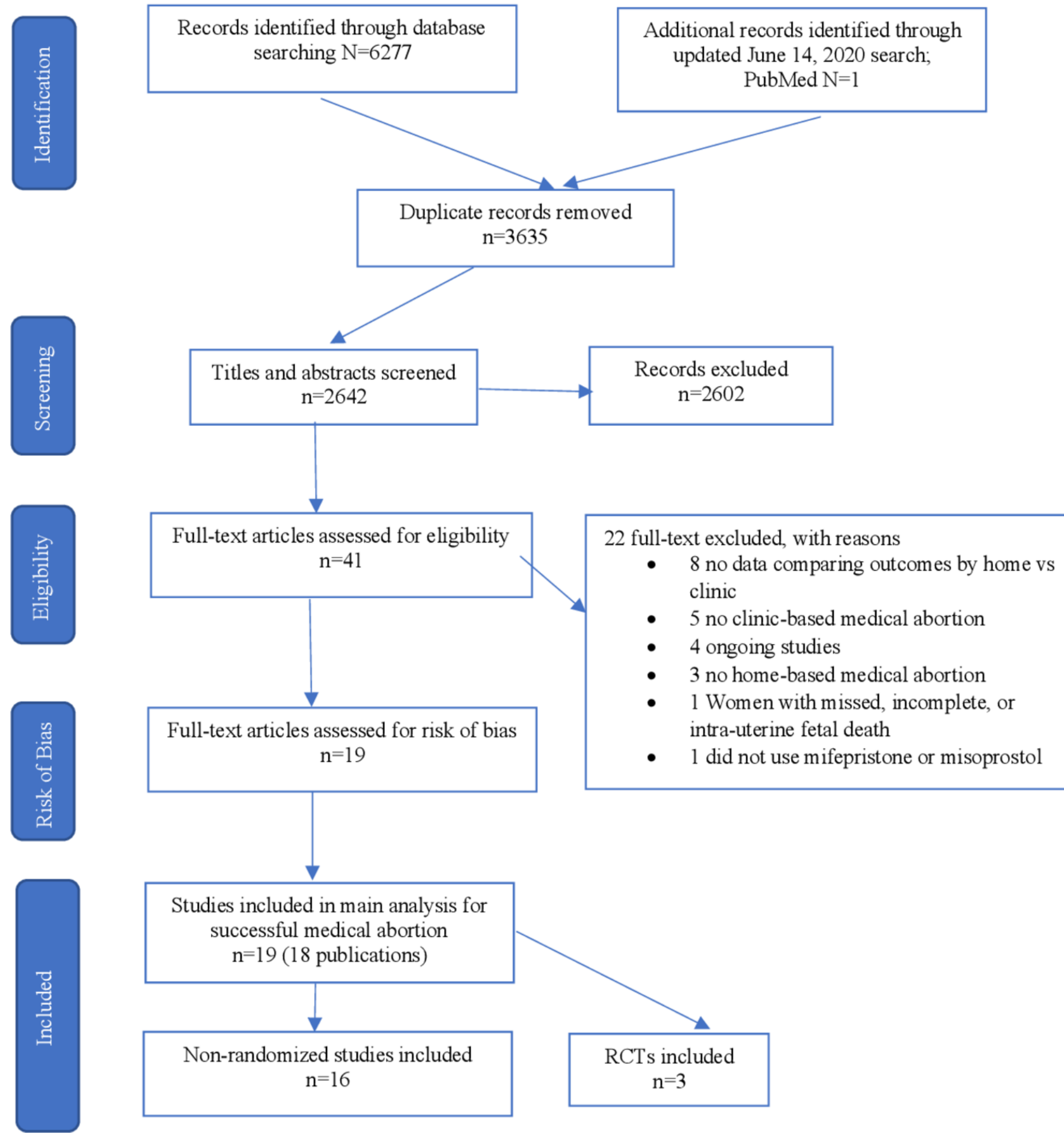

Figure 1 Study selection, RCTs and NRSs. RCTs, randomised controlled trials.

participant characteristics at baseline (online supplemental table 1).

All studies used a combination regimen that involved the administration of mifepristone and misoprostol. All studies reported that $200 \mathrm{mg}$ of oral mifepristone was administered by a provider in the clinic or hospital, and that 24-48 hours later $400-800 \mu \mathrm{g}$ of misoprostol was selfadministered by the woman at home (intervention group) or by a provider in a clinic (comparison group) orally, buccally, vaginally or sublingually. Thus, in all included studies, at least one stage of the process was supervised by a provider and took place in a clinic. Specific details on the medication abortion regimens used in included studies are provided in online supplemental table 1.

Studies conducted follow-up with participants 10-20 days after mifepristone administration ${ }^{17}$ 22 24-27 29-36 or after the completion of post-treatment menstruation. ${ }^{21}{ }^{23}$ Evidence from NRSs that reported on compliance showed no significant differences in compliance with the medical abortion protocol between the two 
Table 1 Study characteristics

\begin{tabular}{|c|c|c|c|c|c|}
\hline \multirow[b]{2}{*}{ Study } & \multirow[b]{2}{*}{ Location } & \multirow[b]{2}{*}{ Study type } & \multicolumn{2}{|c|}{ No of women recruited (lost to follow-up) } & \multirow{2}{*}{$\begin{array}{l}\text { Successful abortion RR } \\
(95 \% \mathrm{Cl})\end{array}$} \\
\hline & & & Home based (H) & Clinic based (C) & \\
\hline Akin et al $2004^{24}$ & Turkey & NRS & $104(4)$ & $104(3)$ & $1.11(0.98$ to 1.25$)$ \\
\hline Alam et al $2018^{26}$ & Bangladesh & NRS & $1619(2)$ & $125(4)$ & $1.01(0.97$ to 1.04$)$ \\
\hline Bracken et al $2006^{27}$ & Albania & NRS & $361\left(5^{\star \star}\right)$ & $48(1)$ & $0.99(0.95$ to 1.04$)$ \\
\hline Bracken $2010^{28}$ & India & NRS & $530(21)$ & $69(3)$ & $0.96(0.89$ to 1.04$)$ \\
\hline Elul et al $2001^{35}$ & Vietnam & NRS & $106(8)$ & $14(0)$ & 1.22 (.93 to 1.61$)$ \\
\hline Elul et al $2001^{35}$ & Tunisia & NRS & $170(4)$ & $25(0)$ & $1.06(0.91$ to 1.23$)$ \\
\hline Hajri et al $2004^{29}$ & Tunisia & NRS & $252(9)$ & $82(0)$ & $1.04(0.98$ to 1.11$)$ \\
\hline lyengar et al $2016^{30}$ & India & NRS & 342 (not reported) & 389 (not reported) & $1.00(0.96$ to 1.04$)$ \\
\hline Karki et al $2009^{31}$ & Nepal & NRS & $323(31)$ & $77(2)$ & $0.63(0.30$ to 1.34$)$ \\
\hline Provansal et al $2009^{16}$ & France & NRS & $143(30)$ & $162(64)$ & 0.91 (0.84 to 0.97$)$ \\
\hline Raghavan et al $2012^{33}$ & Vietnam & NRS & 1933 (not reported) & 366 (not reported) & $1.02(0.99$ to 1.04$)$ \\
\hline Shrestha and Sedhai $2014^{22}$ & Nepal & $\mathrm{RCT}$ & $94(2)$ & $94(2)$ & $1.02(0.92$ to 1.14$)$ \\
\hline Shuchita et al $2008^{34}$ & India & NRS & $76(1)$ & $23(1)$ & 0.99 (0.89 to 1.10$)$ \\
\hline Song et al $2018^{23}$ & China & $\mathrm{RCT}$ & $283(38)$ & $250(29)$ & 0.99 (0.95 to 1.03 \\
\hline
\end{tabular}

** Represents disaggregated loss-to follow-up (follow-up among both clinicand home users)

NRS, non-randomised studies; RCTs, randomised controlled trials; RR, risk ratio.

groups. Three studies 252628 found that the average proportion of home-based participants who completed the medical abortion regimen perfectly was $98.3 \%$ (2642/2687), compared with $98.0 \%(295 / 301)$ of women in the provider-administered group. Similarly, four studies 25263435 reported that only $1.1 \%$ (26/2339) of women in the home-based group reported not taking the misoprostol on time, compared with $1.9 \%(5 / 269)$ in the clinic-based group. In the home-based group, an average of $1.5 \%(41 / 2687)$ did not return to the clinic for follow-up, compared with $3.0 \%$ (9/301) of women in the clinic-based group. ${ }^{25} 2628$ Data from 12 NRSs found that, on average, $77.3 \%(4377 / 5600)$ of home-based participants had companionship during their abortion. 1617 24-26 28 30-32 34-36

\section{Risk of bias}

We assessed the risk of bias for our primary outcome of successful abortion using GRADE criteria. We graded the certainty of the evidence for the three RCTs to be moderate. We downgraded the certainty due to high risk of bias. The $\mathrm{RCT}^{21-23}$ were classified as unclear risk of bias (online supplemental table 2). We did not assess publication bias for the success of medical abortion for the RCTs given there are only three studies.

For the 16 NRSs (all prospective cohort studies), we graded the certainty of the evidence for the outcome of successful abortion to be very low. We downgraded the findings by one level from low certainty of evidence due to high risk of bias (online supplemental table 3). We rated all 16 prospective cohort studies at serious risk of bias because we judged at least one 'risk of bias' domain to be at serious risk of bias. All studies had a serious risk of bias in the same domains: bias due to measurement of outcomes and bias due to confounding. There was bias in measurement of outcomes because they were measured by assessors aware of whether participants had administered misoprostol at home or at the clinic. There was a serious risk of bias due to confounding because the studies were not randomised, and we do not know whether gestational age (a known confounder) had an independent effect on the outcome. In addition to bias due to measurement of outcomes and bias due to confounding seen in the other studies, we classified Provansal et $a l^{16}$ and Okonofua $e t a l^{36}$ to have a serious risk of bias due to missing data because outcome data were not available for over $20 \%$ of the participants.

\section{Successful abortion}

Data collected from 11576 participants in 3 RCTs and 16 NRSs were included in our main effect analysis that evaluated the comparative success of home-based and clinic-based administration of medical abortion among women seeking abortion. Among 1452 participants, the three RCTs $^{21-23}$ found no difference between homebased and clinic-based administration of medical abortion in having a successful abortion (RR 0.99, 95\% CI 0.98 to 1.01 ; figure 2 ). Of the home-based participants, $95.3 \%(705 / 740)$ had a successful abortion, compared with $95.8 \%(682 / 712)$ in the clinic-based group. Similarly, across 10124 participants in 16 NRSs there was no evidence of a difference in successful abortion between 


\begin{tabular}{|c|c|c|c|c|}
\hline & Home & Clinic & Risk ratio & Risk ratio \\
\hline Study or Subgroup & Events Total & Events Total & Weight $\mathrm{M}-\mathrm{H}$, Random, $95 \% \mathrm{Cl}$ & M-H, Random, $95 \% \mathrm{Cl}$ \\
\hline
\end{tabular}

\begin{tabular}{lrrrrrrr|l}
\hline Li 2017 & 357 & 365 & 365 & 370 & $79.7 \%$ & $0.99[0.97,1.01]$ & \\
Shrestha 2014 & 82 & 92 & 80 & 92 & $2.7 \%$ & $1.02[0.92,1.14]$ \\
Song 2018 & 266 & 283 & 237 & 250 & $17.6 \%$ & $0.99[0.95,1.03]$ \\
& & & & & & & &
\end{tabular}

Test for subgroup differences: Not applicable

Figure 2 Forest plot: successful abortion, RCTs. M-H, Mantel-Haenszel; RCTs, randomised controlled trials.

home-based and clinic-based participants (RR 0.99, 95\% CI 0.97 to 1.01; figure 3). Evidence from the NRSs indicates that $93.8 \%(7559 / 8062)$ of home-based participants had a successful abortion compared with $94.0 \%$ (1939/2062) of clinic participants. The rates of successful abortion in the NRSs ranged from $86.7 \%(124 / 143)^{16}$ to $97.2 \%(345 / 355)^{27}$ in the home-based group, and from $78.6 \%(11 / 14)^{35}$ to $100 \%(32 / 32)^{36}$ among the clinicbased group.

The certainty of the evidence for successful abortion ranged from very low (NRSs) to moderate (RCTs). The certainty of the evidence for the 16 NRS was downgraded from low to very low due to high risk of bias and publication bias. The certainty of the evidence for the three RCTs was downgraded from moderate by one level for high risk of bias.

We completed meta-analysis on 16 NRSs and 3 RCTs with dichotomous prescribing outcomes. For our metaanalysis on successful abortion, we did not detect serious heterogeneity $\left(\mathrm{I}^{2}=52 \%\right.$ for 16 NRSs and $\mathrm{I}^{2}=0 \%$ for three RCTs). We did not assess the RCTs for heterogeneity because only three studies were included.

\section{Side-Effects}

Side effects were reported inconsistently across studies, either as dichotomous or continuous measures. We did

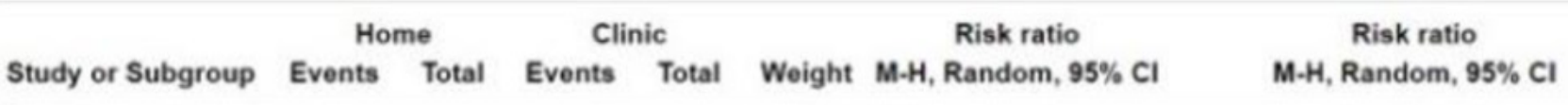

\begin{tabular}{|c|c|c|c|c|c|c|c|c|}
\hline Elul 2001 - Tunisia & 158 & 170 & 22 & 25 & $1.7 \%$ & $1.06[0.91,1.23]$ & - & ـ \\
\hline Elul 2001 - Vietnam & 102 & 106 & 11 & 14 & $0.6 \%$ & $1.22[0.93,1.61]$ & & \\
\hline Dagousset 2004 (1) & 114 & 120 & 286 & 289 & $9.0 \%$ & $0.96[0.92,1.00]$ & $\bullet$ & \\
\hline Bracken 2010 & 453 & 509 & 61 & 66 & $5.0 \%$ & $0.96[0.89,1.04]$ & $\rightarrow$ & \\
\hline Alam 2018 (2) & 1558 & 1617 & 116 & 121 & $9.7 \%$ & $1.01[0.97,1.04]$ & & \\
\hline Bracken 2006 & 345 & 355 & 46 & 47 & $8.5 \%$ & $0.99[0.95,1.04]$ & & \\
\hline Akin 2004 & 92 & 104 & 83 & 104 & $2.5 \%$ & $1.11[0.98,1.25]$ & & \\
\hline Alam 2013 (2) & 485 & 520 & 98 & 109 & $5.8 \%$ & $1.04[0.97,1.11]$ & & - \\
\hline Hajri 2004 & 233 & 241 & 76 & 82 & $6.0 \%$ & $1.04[0.98,1.11]$ & & - \\
\hline lyengar 2016 & 308 & 327 & 352 & 373 & $9.9 \%$ & $1.00[0.96,1.04]$ & & \\
\hline Karki 2009 & 267 & 292 & 68 & 75 & $4.6 \%$ & $1.01[0.93,1.09]$ & & \\
\hline Ngoc 2004 (3) & 1231 & 1390 & 164 & 174 & $9.2 \%$ & $0.94[0.90,0.98]$ & $\rightarrow$ & \\
\hline Okonufua 2014 (4) & 152 & 159 & 32 & 32 & $7.3 \%$ & $0.97[0.92,1.02]$ & $\rightarrow$ & \\
\hline Provansal 2009 & 124 & 143 & 155 & 162 & $5.3 \%$ & $0.91[0.84,0.97]$ & $\rightarrow$ & \\
\hline Raghavan 2012 (5) & 1865 & 1933 & 347 & 366 & $11.8 \%$ & $1.02[0.99,1.04]$ & & - \\
\hline Shuchita 2008 & 72 & 76 & 22 & 23 & $3.2 \%$ & $0.99[0.89,1.10]$ & $\rightarrow$ & \\
\hline Total $(95 \%$ Cl) & & 8062 & & 2062 & $100.0 \%$ & $0.99[0.97,1.01]$ & 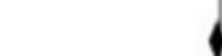 & \\
\hline Total events: & 7559 & & 1939 & & & & & \\
\hline \multicolumn{7}{|c|}{$\begin{array}{l}\text { Heterogeneity: Tau }=0.00 ; C h i^{2}=31.45, d f=15(P=0.008) ; l^{2}=52 \% \\
\text { Test for overall effect: } Z=0.72(P=0.47)\end{array}$} & $\begin{array}{r}0.7 \quad 0.85 \\
\text { Favors clinic }\end{array}$ & $\begin{array}{l}1 \cdot 2 \quad 1.5 \\
\text { Favors home }\end{array}$ \\
\hline
\end{tabular}

Figure 3 Forest plot: successful abortion, NRSs. Published data from Ngo 2011, unpublished data received from trialist, unpublished data received from $\mathrm{Ngo} 2011$, unpublished data received from trialist, unpublished data received from trialist. $\mathrm{M}-\mathrm{H}$, Mantel-Haenszel; NRSs, non-randomised studies. 
not run meta-analyses of continuous measures because an insufficient number of studies reported on these outcomes. Side effects analysed in this review included pain/cramps, vomiting, fever/chills, nausea and heavy bleeding. Table 2 provides data on the proportion of participants in each group who reported side effects, and the duration of side effects by group.

Analyses of dichotomous measures showed no significant difference in the occurrence of any side effects between home-based and clinic-based participants, and the reported duration of all side-effects were comparable between the two groups.

The average observed rate of pain/cramps among homebased women was $60.0 \%(389 / 648)$ in RCTs and $31.4 \%$ $(163 / 519)$ in NRSs, compared with $59.0 \%(366 / 620)$ and $12.9 \%$ (50/386) of clinic-based participants in RCTs and NRSs, respectively. ${ }^{21} 23303436$ Among these studies, ${ }^{30}$ reported on severe abdominal pain and Shrestha and Sedhai reported on acceptable cramping. The mean rate of vomiting was $5.4 \%(40 / 740)$ for home-based RCT participants, $28.5 \%(676 / 2366)$ for home-based NRS participants, 5.6\% (40/712) for clinic-based RCT participants and $24.7 \%$ (70/283) for clinic-based NRS participants. ${ }^{21-2325263436}$ An average of 25.5\% (189/740) and $59.1 \%(1563 / 2645)$ of home-based participants in the RCTs and NRSs, respectively, reported nausea, compared with $24.7 \%(176 / 712)$ and $58.7 \%$ (182/310) of RCT and NRS clinic-based participants. ${ }^{21-23} 25-273436$ No RCTs reported dichotomous measures of fever/chills. The average observed rate of fever/chills in NRSs was $37.6 \%$ (803/2136) among women in the home-based group, and $16.0 \%(81 / 507)$ among women in the clinicbased group. ${ }^{25} 30{ }^{34}$ Similarly, only NRSs reported heavy bleeding dichotomously, and the average observed events of heavy bleeding in these studies was 22.0\% (584/2656) among women in the home group, compared with $20.9 \%$ $(129 / 616)$ in the provider-administered group. ${ }^{25} 26303436$

Across all studies, only two NRSs reported complications requiring hospitalisation; three participants from the home-based administration group and no participants from the clinic-based administration group reported complications requiring hospitalisations. ${ }^{17} 26$ Analysis showed no statistically significant difference in having any complication requiring hospitalisation between the two groups (RR $1.58,95 \%$ CI 0.08 to 29.81 ).

\section{Acceptability}

Most studies reported on at least one acceptability measure. All three RCTs only reported on whether participants were satisfied or highly satisfied with the treatment. Studies that reported only proportions or provided events with no denominator and did not include enough information for the study authors to calculate the denominator are excluded from analysis, but data on acceptability from these studies is included in table 3 .

Medication abortion was highly acceptable to women in both the home-based and clinic-based groups. Ninetyfour per cent $(94.6 \% ; 700 / 740)$ of participants from
RCTs and $90.8 \%(4775 / 5258)$ of participants from NRSs who had home-based medical abortions reported being satisfied or highly satisfied with the method, compared with $94.5 \%(673 / 712)$ of clinic-based RCT participants and $89.4 \%(1256 / 1405)$ of clinic-based NRS participants. ${ }^{161721-23252628-323436}$ Data from NRSs indicate that a higher proportion of participants in the home-based medical abortion group indicated that they would select the medical abortion method again compared with the clinic group; $83.5 \%$ (2137/2560), compared with 53.6\% (512/955), though these differences were not significant. ${ }^{16} 1726303136$ Approximately 93\% (2369/2258) of home-based NRS participants indicated that they would select home use for future medical abortion, compared with $33.1 \%$ (345/1043) of NRS participants in the clinicbased group. ${ }^{16} 1725303236$ Only 4.1\% (95/2337) of NRS participants in the home-based group $(n=2337)$ indicated they would select clinic use for future medical abortion, compared with $64.7 \%(415 / 641)$ of clinic-based NRS participants. ${ }^{25} 303236$ An average of $87.22 \%$ (2231/2558) of home-based NRS participants would recommend the medical procedure to a friend for a future abortion compared with $52.67 \%(503 / 955)$ of NRS women in the clinic-based group. ${ }^{161726303136}$

Meta-analysis did not reveal a difference between the two groups in the level of satisfaction with the procedure (risk difference (RD) $0.01,95 \% \mathrm{CI}-0.03$ to 0.05 ) probability of choosing the medical abortion method again (RD $0.02,95 \% \mathrm{CI}-0.04$ to 0.09 ), or the probability of recommending the medical abortion procedure to a friend ( $\mathrm{RD}$ $0.06,95 \% \mathrm{CI}-0.04$ to 0.15 ). However, the meta-analysis did provide evidence of a significant difference between the two groups in the probability of selecting homebased abortion for future medical abortion between the groups (RD $0.50,95 \% \mathrm{CI} 0.32$ to $0.67 ; \mathrm{I}^{2}=96 \%$ ) and selecting clinic-based abortion for future medical abortion between these groups ( $\mathrm{RD}-0.58,95 \% \mathrm{CI}-0.62$ to $-0.54)$. For both of these significant findings, participants were statistically more likely to select the same abortion location they had experienced in the study.

\section{DISCUSSION}

This systematic review and meta-analysis demonstrates that home-based medical abortion, as practiced in the included studies is effective, safe and acceptable to women. This review provides an important update to Ngo's 2011 review, incorporating ten new studies, including three RCTs, that provides a new body of evidence illustrating that there is no difference in the safety, effectiveness, and acceptability between home-based versus clinic-based medical abortion. ${ }^{14}$ This body of evidence comes at a time of global pandemic, when providing access to homebased administration of medical abortion is a key strategy to protect access to abortion, reduce the transmission of COVID-19, and alleviate overburdened healthcare facilities and providers. 


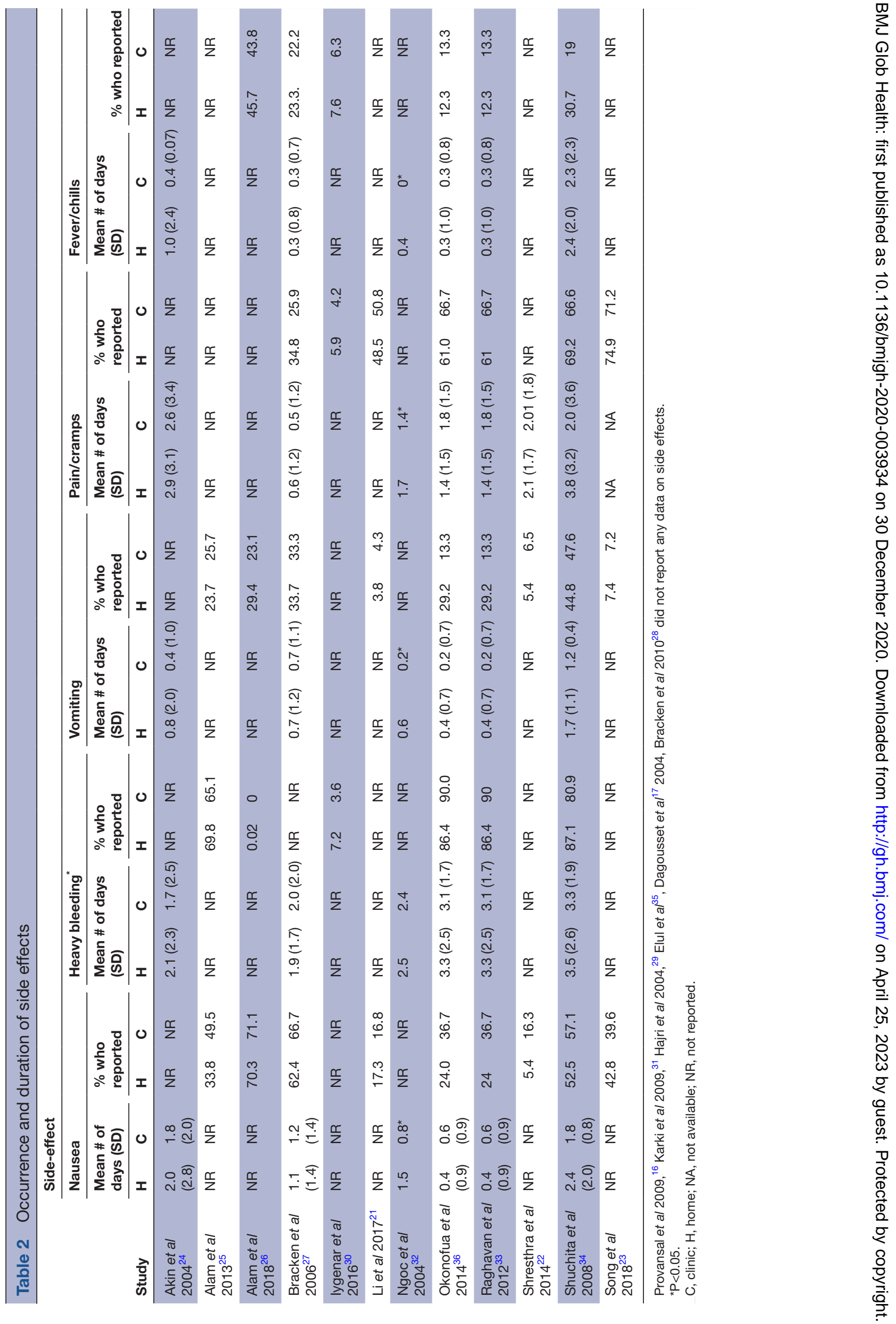




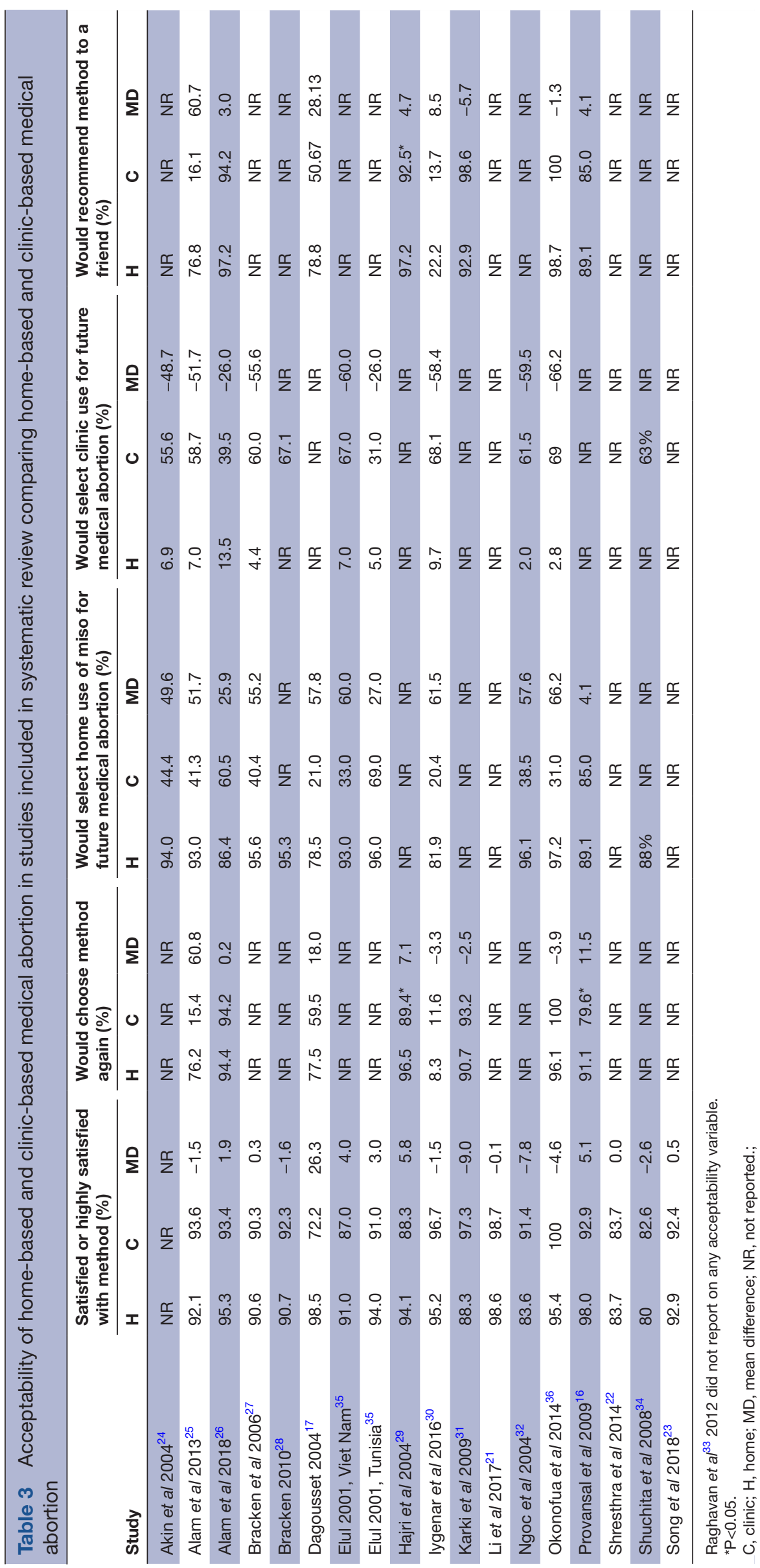


Evidence from the 3 RCTs combined with findings from 16 NRSs show that the effectiveness of medical abortion was high $(>90 \%)$ across studies and that there were no statistically significant differences in the proportion of successful abortion between women who administered their medical abortions at home compared with those who were administered medications at the clinic. The overall certainty of the evidence was moderate. However, this is in part due to the fact that the GRADE criteria is designed to evaluate RCTs whereas our review includes only three RCTs compared with 16 NRSs.

In all studies and in both groups, serious complications related to medical abortion were rare. Analyses showed no differences in the rates of complications requiring hospitalisations between home-based and clinic-based participants. Side effects related to medical abortion were more common, but evidence on the rates of side effects in both groups indicate that differences between the two groups were not statistically significant. Rates of side effects and complications were consistent with those expected by the WHO guidelines and corroborate findings from previous studies. ${ }^{14} 153738$ Additionally, rates of compliance with the medical abortion protocol and follow-up were higher with women who administered at home; findings that should help assuage concerns about compliance and follow-up among those who administer misoprostol at home. Of note, the mifepristone-in-clinic-misoprostol-at-home regimen used by all participants, a regimen classified as home based in our study, has become common practice in some settings, and emerging evidence suggests that the entire mifepristone-misoprostol medical abortion regimen can also be safely and acceptably completed by women at home. ${ }^{39-41}$

This review documents general satisfaction with homebased care and a high likelihood that home-based participants would select home administration for a future abortion medical abortion. These findings support existing evidence that women are satisfied with, and may prefer home-based care for reasons related to convenience, privacy, and access to a support person. ${ }^{42}$ Additionally, people's need or preference for home-based care may only increase in light of the COVID-19 pandemic as the cost and time associated with travelling to seek an abortion was a major barrier to abortion care worldwide prior to the outbreak, particularly in areas with legal although restricted access, ${ }^{43}$ and the reduction of travel associated with home-based care is one documented reason that people may need or prefer this option. ${ }^{344}$

This review has limitations and potential biases. The gestational age for participants from studies included in our review range from 35 to 63 days. Thus, the experiences of people seeking medical abortion beyond 9 weeks of gestation are not represented in this review; however, a recently published paper demonstrated that prospective studies of home-based medical abortion up to 84 days are feasible. ${ }^{45}$ All studies in this review used a combined mifepristone and misoprostol drug regimen and results are therefore not applicable to scenarios where mifepristone is unavailable, or where misoprostol is used in higher doses. Finally, all women included in the studies engaged with and had access to clinic services at some point in their abortion process, and thus results are not applicable to settings where people have no interaction with formal healthcare services. Future studies should compare mifepristone and misoprostol administration or misoprostol only at home to mifepristone in clinic. Future research should also explore the efficacy, safety, and acceptability of home administration of medical abortion among people with pregnancies beyond 9weeks of gestation, among those utilising a misoprostol-alone regimen, and those self-managing their abortions without in-person provider supervision, including those accessing abortifacients online or through pharmacies. Finally, further research is needed on subgroups who face systematic barriers to abortion access and therefore are at an increased risk for unsafe abortion, including adolescents in the Global South, adolescents and widowed and divorced people in humanitarian contexts, and those living in settings with restrictive abortion laws.

The majority of the data presented in this review were from NRSs, and results are thus inherently prone to selection bias. ${ }^{46}$ Women in these studies were able to choose between home-based and clinic based methods, and it is possible that, for example, women who were affected by stigma, concerned about concealing their abortion, or did not have a comfortable home environment would be more likely to select the clinic-based method. ${ }^{47} 48$ The ability of the participant to select a method is an important ethical attribute of studies on abortion given the ethical issues inherent in denying people their choice in method, but this study design does contribute to risk of bias. Our review is also limited by the fact that no studies blinded the outcome assessor, methods for determining successful abortion varied across studies, and only one study reported controlling for confounding factors, including gestational age. Future studies should blind the outcome assessor to reduce performance bias and analyses should control for gestational age, a known confounder.

\section{CONCLUSION}

The COVID-19 pandemic creates both the necessity and opportunity to innovate to meet people's essential sexual and reproductive needs. These innovations must be integrated into long-term service delivery and policy strategies to expand abortion service delivery options in the post-COVID era. It is imperative that researchers, policymakers and practitioners continue to develop, evaluate and expand access to novel and innovative service delivery models such as telemedicine for medical abortion, pharmacist and community health worker provision of medical abortion, and self-management of medical abortion with hotline support. ${ }^{5}{ }^{49-52}$ Policy-makers should consider the new body of evidence presented in this review alongside national and international guidelines on medical abortion 
to update their clinical guidelines and policies that expand access to home-based abortion care.

Offering people at or below 9 weeks of gestation with access to all necessary information and support, and providing them with the option to return to the clinic to take misoprostol, or administer the drug at home will not only protect access to safe, time-sensitive abortion care, but will also reduce the number and cost of clinic visits, and relieve the strain on already overburdened healthcare systems worldwide.

Twitter Katherine Gambir @KatherineGambir

Acknowledgements We thank the authors who provided responses to our questions about their studies. We also thank Mary Chu, Population Council librarian, for supporting us on the search strategy.

Contributors CG drafted the protocol with input from KG and TDN. KG developed and ran the search strategy. KG, KAN and CG obtained copies of the studies. KG, KAN and CG selected which studies to include. KG, KAN and CG extracted data from studies and entered data into RevMan Web Version. KG and KAN assessed risk of bias and certainty of evidence for the primary and secondary outcomes in each study. KG conducted the analysis with input from KAN, CG and TDN. CG interpreted the analysis and drafted the final review with support from KG and TDN.

Funding The systematic review and meta-analysis were supported by funding from the William \& Flora Hewlett Foundation.

Disclaimer The findings and conclusions presented in this paper are those of the authors and do not necessarily reflect the official position of William \& Flora Hewlett Foundation.

\section{Competing interests None declared.}

Patient consent for publication Not required.

Provenance and peer review Not commissioned; externally peer reviewed.

Data availability statement Data are available on request. We permit the sharing of data from this research article. All data published in this article are available on written request to authors.

Supplemental material This content has been supplied by the author(s). It has not been vetted by BMJ Publishing Group Limited (BMJ) and may not have been peer-reviewed. Any opinions or recommendations discussed are solely those of the author(s) and are not endorsed by BMJ. BMJ disclaims all liability and responsibility arising from any reliance placed on the content. Where the content includes any translated material, BMJ does not warrant the accuracy and reliability of the translations (including but not limited to local regulations, clinical guidelines, terminology, drug names and drug dosages), and is not responsible for any error and/or omissions arising from translation and adaptation or otherwise.

Open access This is an open access article distributed in accordance with the Creative Commons Attribution Non Commercial (CC BY-NC 4.0) license, which permits others to distribute, remix, adapt, build upon this work non-commercially, and license their derivative works on different terms, provided the original work is properly cited, appropriate credit is given, any changes made indicated, and the use is non-commercial. See: http://creativecommons.org/licenses/by-nc/4.0/.

Author note We acknowledge that not all people who seek and receive abortion care identity as women. However, existing research on this topic specifically refers to women, and our review includes studies that only recruited women to participate. Thus, throughout this paper, we use the words 'woman/women' and the pronouns 'she/her' when discussing our data in the studies reviewed. We use the more gender-inclusive term 'people' in all other instances.

\section{ORCID iD}

Katherine Gambir http://orcid.org/0000-0003-3491-0582

\section{REFERENCES}

1 Ganatra B, Gerdts C, Rossier C, et al. Global, regional, and subregional classification of abortions by safety, 2010-14: estimates from a Bayesian hierarchical model. The Lancet 2017;390:2372-81.

2 Singh S, Remez L, Sedgh G, et al. Abortion worldwide 2017: Uneven Progress and Unequal Access. N Y Guttmacher Inst.
3 Berer M. Medical abortion: issues of choice and acceptability. Reprod Health Matters 2005;13:25-34.

4 Barot S. The roadmap to safe abortion worldwide: lessons from new global trends on incidence, legality and safety. N Y Guttmacher Inst 2018;21:6.

5 Footman K, Keenan K, Reiss K, et al. Medical abortion provision by pharmacies and drug sellers in low- and middle-income countries: a systematic review. Stud Fam Plann 2018;49:57-70.

6 Yarnall J, Swica Y, Winikoff B. Non-Physician clinicians can safely provide first trimester medical abortion. Reprod Health Matters 2009;17:61-9.

7 Guttmacher Institute. Medication abortion restrictions burden women and Providers - and threaten U.S. trend toward very early abortion, 2013. Available: https://www.guttmacher.org/gpr/2013/03/ medication-abortion-restrictions-burden-women-and-providers-andthreaten-us-trend-toward [Accessed 14 Apr 2020].

8 World Health Organization. Safe abortion: technical and policy guidance for health systems evidence summaries and grade tables. World Health Organization, 2012.

9 Shannon C, Winikoff B. How much supervision is necessary for women taking mifepristone and misoprostol for early medical abortion? Womens Health 2008;4:107-11.

10 World Health Organization (WHO). Shortage of personal protective equipment endangering health workers worldwide: who 2020.

11 Schwirtz M. Nurses die, doctors fall sick and panic rises on virus front lines. the new York times, 2020. Available: https://www. nytimes.com/2020/03/30/nyregion/ny-coronavirus-doctors-sick.html [Accessed 14 Apr 2020].

12 Cavallo JJ, Donoho DA, Forman HP. Hospital capacity and operations in the coronavirus disease 2019 (COVID-19) PandemicPlanning for the Nth patient. American Medical Association 2020:e200345.

13 Ho PC. Women's perceptions on medical abortion. Contraception 2006;74:11-15

14 Ngo TD, Park MH, Shakur H, et al. Comparative effectiveness, safety and acceptability of medical abortion at home and in a clinic: a systematic review. Bull World Health Organ 2011;89:360-70.

15 Gambir K, Kim C, Necastro KA, et al. Self-Administered versus provider-administered medical abortion. Cochrane Database Syst Rev 2020:3:CD013181.

16 Provansal M, Mimari R, Grégoire B, et al. [Medical abortion at home and at hospital: a trial of efficacy and acceptability]. Gynecol Obstet Fertil 2009;37:850-6.

17 Dagousset I, Fourrier E, Aubény E, et al. [Use of Misoprostol for medical abortion: a trial of the acceptability for home use]. Gynecol Obstet Fertil 2004;32:28-33.

18 Gambir K, Garnsey C, Necastro KA, et al. Effectiveness, safety, and acceptability of medical abortion at home versus in the clinic: an updated systematic review in response to COVID-19 2020.

19 BMJ Best Practice. What is grade? Available: https://bestpractice. bmj.com/info/us/toolkit/learn-ebm/what-is-grade/ [Accessed $24 \mathrm{Apr}$ 2020].

20 The Cochrane Collaboration. Review manager web (RevMan web.) 2019.

21 Li C-L, Song L-P, Tang S-Y, et al. Efficacy, safety, and acceptability of low-dose mifepristone and self-administered misoprostol for ultraearly medical abortion: a randomized controlled trial. Reprod Sci 2017;24:731-7.

22 Shrestha A, Sedhai LB. A randomized trial of hospital vs home self administration of vaginal misoprostol for medical abortion. Kathmandu Univ Med J 2014;12:185-9.

23 Song L-P, Tang S-Y, Li C-L, et al. Early medical abortion with self-administered low-dose mifepristone in combination with misoprostol. J Obstet Gynaecol Res 2018;44:1705-11.

24 Akin A, Blum J, Özalp S, et al. Results and lessons learned from a small medical abortion clinical study in turkey. Contraception 2004;70:401-6.

25 Alam A, Bracken H, Johnston HB, et al. Acceptability and feasibility of mifepristone-misoprostol for menstrual regulation in Bangladesh. Int Perspect Sex Reprod Health 2013;39:79-87.

26 Alam A, Lotarevich T, Das TR, et al. Mifepristone-misoprostol for menstrual regulation in public sector facilities in Bangladesh. Int $J$ Gynaecol Obstet 2018;140:205-10.

27 Bracken $\mathrm{H}$, Gliozheni O, Kati K, et al. Mifepristone medical abortion in Albania: results from a pilot clinical research study. Eur $J$ Contracept Reprod Health Care 2006;11:38-46.

28 Bracken H. Family Planning Association of India (FPAl)/Gynuity Health Projects Research Group for Simplifying Medical Abortion in India. Home administration of misoprostol for early medical abortion in India. Int J Gynecol Obstet 2010;108:228-32. 
29 Hajri S, Blum J, Gueddana N, et al. Expanding medical abortion in Tunisia: women's experiences from a multi-site expansion study. Contraception 2004;70:487-91.

30 lyengar K, Klingberg-Allvin M, lyengar SD, et al. Home use of misoprostol for early medical abortion in a low resource setting: secondary analysis of a randomized controlled trial. Acta Obstet Gynecol Scand 2016;95:173-81.

31 Karki C, Pokharel H, Kushwaha A, et al. Acceptability and feasibility of medical abortion in Nepal. Int J Gynecol Obstet 2009;106:39-42.

32 Ngoc NTN, Nhan VQ, Blum J, et al. Is home-based administration of prostaglandin safe and feasible for medical abortion? results from a multisite study in Vietnam. BJOG Int J Obstet Gynaecol 2004;111:814-9.

33 Raghavan S, Ngoc NthiN, Shochet T, et al. Clinic-level introduction of medical abortion in Vietnam. Int J Gynaecol Obstet 2012;119:39-43.

34 Shuchita M, Shveta K, Batya E, et al. Simplifying medical abortion: home administration of misoprostol. J Obstet Gynecol India 2008;58:410-6.

35 Elul B, Hajri S, Ellertson C, et al. Can women in less-developed countries use a simplified medical abortion regimen? The Lancet 2001;357:1402-5.

36 Okonofua F, Shittu O, Shochet T, et al. Acceptability and feasibility of medical abortion with mifepristone and misoprostol in Nigeria. Int J Gynaecol Obstet 2014;125:49-52.

37 WHO. Medical management of abortion. WHO. Available: http:// www.who.int/reproductivehealth/publications/medical-managementabortion/en/ [Accessed 14 Apr 2020].

38 Kruse B, Poppema S, Creinin MD, et al. Management of side effects and complications in medical abortion. Am J Obstet Gynecol 2000;183:S65-75.

39 Chong E, Frye LJ, Castle J, et al. A prospective, non-randomized study of home use of mifepristone for medical abortion in the U.S. Contraception 2015:92:215-9.

40 Louie KS, Tsereteli T, Chong E, et al. Acceptability and feasibility of mifepristone medical abortion in the early first trimester in Azerbaijan. Eur J Contracept Reprod Health Care 2014;19:457-64.
41 Platais I, Tsereteli T, Grebennikova G, et al. Prospective study of home use of mifepristone and misoprostol for medical abortion up to 10 weeks of pregnancy in Kazakhstan. Int J Gynecol Obstet 2016;134:268-71.

42 Moseson H, Herold S, Filippa S, et al. Self-managed abortion: a systematic scoping review. Best Pract Res Clin Obstet Gynaecol 2020;63:87-110.

43 Barr-Walker J, Jayaweera RT, Ramirez AM, et al. Experiences of women who travel for abortion: a mixed methods systematic review. PLoS One 2019;14:14.

44 Fuentes L, Baum S, Keefe-Oates B, et al. Texas women's decisions and experiences regarding self-managed abortion. BMC Womens Health 2020;20:6.

45 Moseson H, Jayaweera R, Raifman S, et al. Self-managed medication abortion outcomes: results from a prospective pilot study. Reprod Health 2020;17:1644 https://doi.org/

46 Reeves BC, Deeks JJ, Higgins J. 13 including non-randomized studies. Cochrane Handb Syst Rev Interv 2008;1:391.

47 Lohr PA, Wade J, Riley L, et al. Women's opinions on the home management of early medical abortion in the UK. J Fam Plann Reprod Health Care 2010;36:21-5.

48 Shellenberg KM, Moore AM, Bankole A, et al. Social stigma and disclosure about induced abortion: results from an exploratory study. Glob Public Health 2011;6:S111-25.

49 Grossman D, Grindlay K. Safety of medical abortion provided through telemedicine compared with in person. Obstet Gynecol 2017;130:778-82.

50 Gerdts C, Hudaya I. Quality of care in a safe-abortion Hotline in Indonesia: beyond harm reduction. Am J Public Health 2016;106:2071-5.

51 Drovetta RI. Safe abortion information hotlines: an effective strategy for increasing women's access to safe abortions in Latin America. Reprod Health Matters 2015;23:47-57.

52 Gerdts C, Jayaweera RT, Kristianingrum IA, et al. Effect of a smartphone intervention on self-managed medication abortion experiences among safe-abortion Hotline clients in Indonesia: a randomized controlled trial. Int J Gynecol Obstet 2020;149:48-55. 